Interfaces

\title{
'Smashing crockery' : objets cassés, objets-déchets dans la poésie de Derek Mahon
}

\section{Marion Naugrette-Fournier}

\section{(2) OpenEdition}

1 Journals

Édition électronique

URL : http://journals.openedition.org/interfaces/898

DOI : 10.4000/interfaces.898

ISSN : 2647-6754

Éditeur :

Université de Bourgogne, Université de Paris, College of the Holy Cross

\section{Édition imprimée}

Date de publication : 15 juillet 2020

Pagination : 89-111

ISSN : 1164-6225

\section{Référence électronique}

Marion Naugrette-Fournier, «'Smashing crockery' : objets cassés, objets-déchets dans la poésie de Derek Mahon », Interfaces [En ligne], 43 | 2020, mis en ligne le 15 juillet 2020, consulté le 29 décembre 2020. URL : http://journals.openedition.org/interfaces/898 ; DOI : https://doi.org/10.4000/interfaces. 898

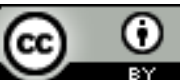

Les contenus de la revue Interfaces sont mis à disposition selon les termes de la Licence Creative Commons Attribution 4.0 International. 


\title{
'SMASHING CROCKERY': OBJETS CASSÉS, OBJETS-DÉCHETS DANS LA POÉSIE DE DEREK MAHON
}

\author{
Marion Naugrette-Fournier \\ Université Sorbonne Nouvelle
}

\begin{abstract}
Résumé : A travers son œuvre le poète nord-irlandais Derek Mahon a fait de la tabula rasa un véritable ars poetica, en utilisant les objets au rebut ou en «cassant» des objets en bon état, pour après les récupérer, voire les recycler en les réintégrant à un nouveau système des objets dont il a établi les règles. Pour ce faire, Mahon n'a cessé de repenser dans ses poèmes la valeur de l'objet au sens économique, métaphysique et poétique du terme, par le biais de la destruction ou de la tabula rasa, conçue comme phase quasi nécessaire et préalable à la revalorisation de l'objet. Par cette revalorisation s'ensuit un processus de reconstruction, où le poète assemble ses « objets transitionnels », pour reprendre les termes de Donald W. Winnicott, comme autant de joujoux qu'il aurait fait siens, afin d'échapper à toute appartenance communautaire, et notamment nord-irlandaise. La poésie de Mahon est ainsi perpétuellement en construction, « A Building Site ».
\end{abstract}

Mots-clés : Mahon (Derek), tabula rasa, objets, objets au rebut, déchets, valeur, destruction, récupération, objets transitionnels, rage for order

\begin{abstract}
Throughout his poetic œuvre the Northern Irish poet Derek Mahon has made the tabula rasa a true ars poetica of his very own, using discarded objects or even 'breaking' objects in good condition, in order to salvage them or recycle them by reintegrating them to a new system of objects of which he alone knows the rules. To achieve such a goal, in his poems Mahon has relentlessly re-envisioned the value of an object economically, metaphysically and poetically, by temporarily destroying it. This 'tabula rasa' phase is thus seen as almost essential to the process of re-evaluating the value of objects, which in turn is followed by a process of reconstructing them, implying the poet's gathering of what Donald W. Winnicott names 'transitional objects'. Mahon's transitional objects, so to speak, act as new toys he has made his own, so that he could be free from any kind of attachment towards a community, and especially a Northern Irish one. Mahon's poetry is thus a 'Building Site' of sorts, always in motion.
\end{abstract}

Keywords: Mahon (Derek), tabula rasa, objects, discarded objects, rubbish, value, destruction, salvaging, transitional objects, rage for order 
Derek Mahon est un poète connu, entre autres, pour son refus d'appartenir à toute communauté, y compris poétique. Dans une lettre ouverte au Irish Times (I6 juillet I987), il affirme ainsi sa volonté de se dissocier de toute appartenance au Belfast Group ${ }^{1}$, fondé par le professeur Philip Hobsbaum en I963 à Belfast, qui fut le lieu de rencontre et de discussions d'une génération émergeante de poètes nord-irlandais, tels que Seamus Heaney, Michael Longley, Paul Muldoon, Edna Longley et parfois Derek Mahon. Le Belfast Group incarnait alors ce qu'on allait ensuite identifier comme l'Ulster Renaissance $^{2}$ des années soixante, qui proposait de transcender les clivages politiques et sociaux de l'Irlande du Nord à cette époque, dans l'axe Dublin-Londres-Belfast.

Ce refus d'être assimilé à un mouvement intellectuel va de pair chez Mahon avec le refus d'appartenir à toute communauté religieuse ou sociale, en commençant par le milieu petit-bourgeois protestant de Glengormley, la banlieue de Belfast d'où il est originaire : « By / Necessity, if not choice, I live here too » (Collected Poems I4) ${ }^{3}$. Mahon n'aura de cesse que de fuir cette identité nord-irlandaise, fuite qui se traduit par un exil physique, notamment en France, en Angleterre et surtout aux États-Unis, mais également par un exil poétique. En effet, au moment où éclatent les Troubles en Irlande du Nord en 1969, Mahon réplique, dans « The Last of the Fire Kings », par ce vers depuis très controversé : « Either way, I am / Through with history » (Poems 1962-1978 64). Sur ce point Mahon adopte une posture radicalement différente de celle de Seamus Heaney, comme il le dit lui-même : "Seamus Heaney, for example, did a lot more digging than I did » (interview avec Eamon Grennan I56), faisant allusion au célèbre poème de Heaney, « Digging » (Heaney 3). Contrairement à Heaney, Mahon se refuse à « creuser » (« digging ») dans les racines historiques et mythologiques du conflit nord-irlandais, préférant aborder l'histoire de manière détournée et se pencher sur une série d'artefacts en fin de course, en fin d'histoire pourrait-on dire, telles les boîtes de conserve dans « The Apotheosis of Tins » :

Deprived of use, we are safe now from the historical nightmare and may give our attention at last to things of the spirit [...]. (CP 69)

Mahon, à un moment crucial de l'histoire de son pays, choisit la neutralité, dans ce qu'il qualifie lui-même de « 'plague on both your houses' attitude », et développe une écriture poétique du détail, qui se veut libérée du « cauchemar de l'Histoire ». Il se concentre sur les objets de la société de consommation - des boîtes en fer-blanc, par exemple - dont il étudie le parcours complet, en commençant par

Sur le Belfast Group et ses origines, voir Matthew Campbell (I00-I07).

Voir Heather Clark, The Ulster Renaissance: Poetry in Belfast 1962-1972 (2006).

Ce recueil sera désigné dans la suite de cet article sous l'abréviation $C P$. 
l'envahissement des objets au sein de notre quotidien et par cette fascination moderne pour l'objet utile, cette « sublimation de l'ustensilité » qu'évoque Barthes dans Mythologies (I42). À la fin du parcours, le poète mahonien " casse », puis « récupère », au sens écologique du terme, ces objets dépossédés de leur statut d' « utensilia », c'est-à-dire d'objets usuels ${ }^{4}$. C'est paradoxalement une fois dépourvus de leur utilité pratique qu'ils acquièrent alors aux yeux du poète leur véritable utilité, c'est-à-dire une utilité poétique, et que commence la quête de la chose que dissimule l'objet; en d'autres termes, «we [...] may give our attention at last / to things of spirit ». Comment alors ce processus de récupération des objets au rebut, échoués par exemple sur une plage ou dans une poubelle, a-t-il pu permettre au poète de créer librement un nouveau système des objets, résolument tourné vers l'extérieur, hors de ce qu'il conviendrait d'appeler la caverne nord-irlandaise, au sens platonicien du terme?

Pour créer un nouveau système des objets, il s'agit d'abord d'en détruire un autre, et c'est cette dialectique entre deux catégories d'objets antagonistes, au cœur de la création poétique mahonienne, que nous nous proposons d'étudier tout d'abord. En effet, Mahon détruit systématiquement la valeur des objets dits « utiles », notamment par le biais de l'ironie. Le poète réévalue ensuite la valeur des objets-déchets et donc les valeurs de la société dont ils sont l'emblème, en commençant par les dévaluer, au sens moral, voire au sens économique du terme. Nous verrons ensuite dans quelle mesure l'esthétique des objets-déchets que développe Mahon tout au long de ses poèmes est tributaire de sa relation œdipienne à sa mère via les objets. À la lumière des analyses du psychanalyste Donald W. Winnicott, nous étudierons alors comment cette pulsion de destruction, que l'on retrouve tout au long de l'œuvre poétique de Mahon, peut trouver une forme de sublimation à travers la destruction des objets, les rendant alors paradoxalement « utilisables » comme le souligne Winnicott dans « Objet de l'usage d'un objet» (Winnicott 2000, I30). Enfin, il s'agira de s'interroger sur les limites de cette pratique de la tabula rasa et de voir comment, afin d'éviter la destruction totale des objets, après une phase de destruction suit une phase de récupération, au cours de laquelle Mahon s'emploie à réutiliser les objets détruits qui s'offrent à lui dans la nature ou sur les terrains vagues. Les objets au rebut deviennent alors des objets transitionnels, permettant au poète de surmonter son traumatisme œedipien et de s'échapper de la caverne nord-irlandaise castratrice.

\section{La revalorisation de l'objet-déchet}

Si la poésie de Derek Mahon est une poésie de l'objet, elle est fondamentalement une poésie des objets au rebut, une apothéose des objets-déchets. Cette préférence pour l'objet au rebut a de multiples 
racines, à la fois psychologiques, voire pathologiques (le refus de l'objet propre qui incarne l'objet maternel), mais aussi historiques et culturelles (la méfiance vis-à-vis de l'éthique protestante de la pureté, susceptible de dissimuler une intolérance vis-à-vis de ce qui fait désordre, ou tout simplement de ce qui est autre). Le déchet présente de nombreux avantages, si l'on peut dire, aux yeux de Mahon d'un point de vue poétique :

- il n'a plus d'étiquette, il est donc anonyme.

- c'est le nom du dé-nommé, comme le dit Barthes dans son essai intitulé « Réquichot et son corps »: «Qu'est-ce que le déchet ? C'est le nom de ce qui a eu un nom, c'est le nom du dé-nommé... » $(\text { (197 })^{5}$. Il n'a plus de fonction utilitaire, on peut donc le réutiliser en le détournant de sa fonction première.

- il est au rebut, il a donc été abandonné et n'appartient plus à aucun maître ou propriétaire: il est libre.

Le déchet est aussi ce dont on dispose, à tous les sens du terme : en anglais, le verbe «dispose » signifie, entre autres, « se débarrasser de », ou bien « jeter » lorsqu'il s'agit de déchets, voire « détruire » ou « éliminer » pour des déchets chimiques ou industriels. À la fin de ce processus qui transforme l'objet en déchet, Mahon peut donc disposer des déchets à sa guise, en éliminer certains mais en garder d'autres, voire les récupérer et même les recycler, en leur donnant une nouvelle vie à travers la voix poétique. Mais ce choix délibéré consistant à réintégrer les déchets dans la chaîne poétique implique une remise en question de la valeur qu'on attribue aux objets, non seulement sur le plan esthétique mais également sur le plan économique. Ce en quoi Mahon ne fait pas figure de cas isolé, mais au contraire se place dans la lignée d'une revendication esthétique et politique, lignée dans laquelle de nombreux artistes ont choisi de s'inscrire depuis le début du vingtième siècle, des surréalistes (avec le readymade de Duchamp) à Damien Hirst et Christian Boltanski plus récemment.

Il y a ainsi une tentative de reclassement des valeurs à l'œuvre dans la poésie de Mahon, où les objets, une fois arrivés au stade de déchets, se reclassent eux-mêmes par l'entremise du poète et déterminent leur propre valeur, comme dans le poème intitulé « The Apotheosis of Tins »: «If we have learnt one thing from our desertion [...] / It is the value of self-definition » (CP 69). L'abandon de l'objet, paradoxalement, permet la redéfinition de sa propre valeur, et ici «desertion » rime avec « self-definition ». Comme le rappelle en effet Georg Simmel dans The Philosophy of Money (1907), la notion de valeur, et notamment la valeur économique, n'est jamais une propriété intrinsèque de l'objet, mais un jugement

Le peintre Bernard Réquichot (I929-I96I) était connu, entre autres, pour sa série des Reliquaires, où il enfermait dans une vitrine des agglomérats de la nature, comme des racines, des débris de feuilles, mais aussi des mottes de terre ou des coquilles d'escargots. 
porté sur cet objet même par les membres d'une société. C'est en particulier le désir (ou la demande en termes économiques), ainsi que la difficulté à acquérir l'objet, qui sont le fondement de sa valeur :

The difficulty of acquisition, the sacrifice offered in exchange, is the unique constitutive element of value, of which scarcity is only the external manifestation, its objectification in the form of quantity. (Simmel I00)

La valeur d'un objet sera donc d'abord déterminée à l'aune de son attractivité, qui est ellemême le fruit d'une construction de ce que Arjun Appadurai, dans son introduction à The Social Life of Things, nomme « a politics of knowledge », un savoir qui est double :

Commodities represent very complex social forms and distributions of knowledge. In the first place, and crudely, such knowledge can be of two sorts: the knowledge (technical, social, aesthetic and so forth) that goes into the prouction of the commodity; and the knowledge that goes into appropriately consuming the commodity. (Appadurai 4I)

Le second type de savoir que décrit Appadurai (« the knowledge that goes into appropriately consuming the commodity ») correspond, entre autres, à la vaste campagne de communication du monde de la publicité, qui fabrique l'objet et le rend désirable, faisant d'un objet insignifiant un objet de consommation à la mode, et donc par là même éminemment convoité.

C'est ce mode de consommation que critique précisément Mahon dans le poème «After the Storm », qui décrit une fois de plus un monde post-apocalyptique dévasté par la tempête, où les objets de consommation se retrouvent balayés par le flot des rivières en crue, déclassés par la liste poétique elle-même où ils se retrouvent juxtaposés les uns à côté des autres :

Detritus of the years, carpet and car, computers and a wide range of expensive gadgetry went spinning down the river with furniture and linen, crockery, shoes and clothes, until it finally gave over; not everyone had full insurance cover. (An Autumn Wind 28-29) ${ }^{6}$ 
Les objets issus de la technologie du monde moderne, tels que les ordinateurs ou autres objets du même ordre, téléphones portables ou tablettes par exemple, qui étaient considérés comme si désirables et donc si coûteux (" computers and a wide range of expensive gadgetry »), subissent un changement de statut dû à la puissance de désordre contenue dans la tempête. Ces objets, véritables prouesses de la technologie, se retrouvent déclassés, tout en bas de l'échelle : d'objets de consommation hautement convoités ils ont été rabaissés au rang de déchets, dans la catégorie infâmante de " detritus of the years », pêle-mêle, avec les tapis, les voitures, les meubles, le linge, la vaisselle (on retrouve encore le mot « crockery » dans l'un des poèmes les plus connus de Mahon, "Courtyards in Delft $»^{7}$ ), les chaussures et les vêtements qui sont emportés par les eaux... On remarquera ici la puissance dévastatrice et déclassante de la tempête - étant un élément naturel, elle est incontrôlable et imprévisible, et apporte, en se déchaînant, non seulement la dévastation mais aussi le désordre, en défaisant ce qui avait été ordonné et en ramenant tous les objets sur un pied d'égalité ${ }^{8}$. On retrouve cette capacité à créer le chaos, caractéristique des forces naturelles, dans plusieurs poèmes de Mahon, comme "The Great Wave », où le poète évoque le passage d'un tsunami se produisant au large de l'Indonésie, à un moment où lui-même se trouvait en Inde à Bangalore. Il y décrit le paysage de désolation et le spectacle des objets de consommation déplacés de leur habitat habituel dans des termes à peu près similaires à ceux de «After the Storm » :

\section{$[\ldots]$}

The swirling mud receded leaving a waste of bodies, furniture, palm trunks, dereliction and in the streets the contents of an ocean.

If 'waste is the new raw material' as they say, our resources are infinite: on black beaches, carrion, groceries, sewage, wide-open fridges ${ }^{9}$

7. Derek Mahon, "Courtyards in Delft” (The Hunt by Night Io). Nous désignerons par la suite ce recueil sous l'abréviation $T H B N$.

8. Ce poème "After the Storm" n'est d'ailleurs pas sans faire penser au célèbre poème de Jonathan Swift (poète que Mahon affectionne tout particulièrement), "A Description of a City Shower" (I7IO), où à la fin du poème le poète décrit les déchets qui envahissent les rues de la ville de Londres sous l'effet de la pluie torrentielle qui s'abat sur la ville: “[...] Sweepings from Butchers Stalls, Dung, Guts, and Blood,/Drown'd Puppies, stinking Sprats, all drench'd in Mud,/Dead Cats and Turnip-Tops come tumbling down the Flood" (Swift 5). Je remercie ici très chaleureusement Marie-Odile Bernez de m'avoir indiqué ce rapprochement très éclairant avec le poème de Mahon.

9. On remarquera ici la façon dont Mahon joue du pentamètre et des accents pour accueillir cet ensemble de déchets, notamment à travers les vers suivants : « carrion, groceries, sewage, wide-open fridges » et « Tractors haul the wreckage, 
Fought over by frenetic gull and crow.

Tractors haul the wreckage, dead car and cow, balconies, splintered bits of bungalow. On the last rock some soapstone Madurai devotional figures... A post-tsunami sky. $(A A W 77)$

Les deux poèmes «After the Storm » et « The Great Wave », qui figurent tous deux dans le recueil An Autumn Wind, se font ainsi écho, l'un étant le pendant de l'autre, décrivant chacun le chaos produit par les éléments (et notamment l'eau). Ce chaos se traduit par le sort dévolu aux objets de consommation, certains de valeur non négligeable sur le plan économique comme ici les réfrigérateurs ou la voiture, qui, devenus méconnaissables et inutilisables, sont relégués au rang de déchets : « wide-open fridges », « dead car» ... Comme nous le faisions remarquer plus haut, la puissance désorganisatrice de la tempête se marque également par le fait que ces objets ont été, par la force des choses, délogés de l'habitat qui était le leur, c'est-à-dire de la maison de leurs propriétaires, et notamment de la cuisine, pour les réfrigérateurs, ou du salon pour ce qui est de la télévision. C'est précisément ce déplacement, voire cette délocalisation, des objets au rebut qui renforce leur caractère de déchet. Un tel déplacement suscite la gêne, voire le dégoût, comme l'observe Michael Thompson dans son ouvrage pionnier sur l'étude des déchets, Rubbish Theory: The Creation and Destruction of Value, qui a grandement influencé Mahon ${ }^{10}$ :

Something which has been discarded, but never threatens to intrude, does not worry us at all. [But rubbish in the wrong place is] emphatically visible and extremely embarrassing. (Thompson 92)

Il est intéressant de constater, dans le cas d'objets comme la télévision, les ordinateurs ou encore les voitures, dans « After the Storm » ou « The Great Wave », que ces objets n'ont pas vocation à devenir déchets, et ont donc été déplacés de manière accidentelle par la tempête ou le tsunami, les rendant inutilisables à cause de la dégradation physique due à l'eau, et précipitant de fait leur transformation d'objets de consommation en objets-déchets.

dead car and cow $»$.

10. «Mahon is clearly familiar with Michael Thompson's provocative Rubbish Theory, an elegant anatomy of rubbish as an essential cultural category. Like Thompson he is interested in the primary relation between rubbish and cultural value, and in particular the process whereby things move from one category to another » (Haughton 326). 
Ce qui est choquant pour le spectateur, c'est bien la vue de ces objets-déchets qui ont envahi l'espace urbain, au lieu de rester à leur place habituelle, au sein de la maison ou des bureaux ${ }^{11}$. Mahon souligne ici la vacuité de l'empire des choses matérielles faisant partie de notre quotidien -en montrant comment les éléments naturels peuvent, en l'espace d'un instant, balayer et renverser les catégories de valeur en réduisant des objets de consommation à la mode, jugés indispensables pour tout foyer, à un amas de déchets déversés pêle-mêle avec des corps d'animaux ou des arbres abattus : «Tractors haul the wreckage, dead car and cow,/balconies, splintered bits of bungalow » («After the Storm », $A A W$ 77). En temps habituel, ce que nous avons déjà trié comme déchet est souvent caché à nos yeux, ou du moins nous sommes-nous arrangés pour que cela soit le cas, pour que les déchets restent à leur place, que cela soit au grenier, à la cave, au fond d'un meuble, ou à la poubelle, dans l'attente d'être définitivement emporté par la collecte d'ordures. Il y a ainsi toute une géographie du déchet, serait-on tenté de dire, à l'œuvre aussi bien à l'intérieur, au sein de nos foyers, qu'à l'extérieur. Il s'agit dans les deux cas de faire disparaître les déchets de notre vue de sorte qu'on les oublie ; on souhaite qu'ils disparaissent sans que l'on s'en aperçoive, de manière souterraine ou invisible, comme le fait remarquer Jean Gouhier dans son remarquable article intitulé « La marge : entre rejet et intégration » :

L'ordure et le déchet suscitent la répulsion et l'expulsion et constituent des marqueurs négatifs des territoires de vie. A l'échelle de l'habitation [...] existent aussi les « bas » et les « lointains » vers lesquels sont repoussés les biens obsolètes, les rebuts, les déchets : le débarras, la cave, le grenier, le pourrissoir, la poubelle. Ils signalent les zones les moins appropriées de l'espace social de vie, les écarts distants du noyau significatif du foyer vers le haut, vers le bas, vers l'extérieur, vers le fonds de la parcelle : ce sont les périphéries et les marges. (Gouhier 8I-82)

De même, Gouhier attire notre attention sur le fait que ce sont surtout dans des espaces en marge de la ville que sont installés, par exemple, les usines de traitement de déchets, ainsi que les rebuts de manière plus générale :

L'histoire nous apprend le rôle de déversoir de certaines périphéries urbaines qui constituent une «zone » déconsidérée où l'on refoule les indésirables, les nuisances, les ordures, les carcasses techniques, les exclus, tous résidus du filtre urbain, épurateur des bonnes valeurs et des belles images de la société. [...]

C'est un cas particulièrement expressif de la fonction d'espace de décharge, de débarras que la banlieue assure pour le compte de la ville-centre. Chaque ville européenne conserve généralement cette

11. Pour une analyse plus « écopoétique » du rôle du déplacement des objets dans la poésie de Mahon, voir NaugretteFournier et Boisseau (I-20). 
attitude traditionnelle assez primaire de rejet hors les murs des nuisances techniques, économiques, sociales, voire politiques, faute d'en assurer une prévention efficace. (Gouhier 8I-82)

Dans le paysage apocalyptique de «The Great Wave » ou d' « After the Storm », Mahon met en scène une inversion des systèmes de répartition de l'espace : ce qui est d'ordinaire contenu à la périphérie se retrouve au centre de l'espace urbain, projeté hors de la cachette où on le relègue d'habitude, visible aux yeux de tous. Si Mahon est un poète de la «rage-for-order » $\left(C P\right.$ 47-48) ${ }^{12}$, n'oublions pas que dans cette expression il y a d'abord «rage » : la rage de la destruction est dans sa poésie un préalable à toute tentative de reconstruction du monde.

\section{Détruire pour mieux reconstruire : la tentation du chaos}

La poésie de Mahon est en effet empreinte d'une fascination pour la destruction, pour l'apocalypse, qu'il considère presque comme un élément purificateur. Le titre même du poème « The Great Wave » fait d'ailleurs peut-être allusion à la célèbre estampe d'Hokusai, Under the Wave off Kanagawa (I826I836), également connue sous le nom de «The Great Wave » (Figure 1, page suivante), où l'on voit une vague gigantesque, comparable à celle d'un tsunami, sur le point de déferler sur une nuée de petits bateaux de pêche, impuissants devant la taille de la vague ${ }^{13}$.

Il y a chez Hokusai comme chez Mahon cette même humilité (de l'artiste) face aux forces terrifiantes de la nature, ainsi qu'une volonté de rappeler à l'homme que ses créations sont impuissantes face à la force de destruction de la nature, et que le système de valeurs qu'il a instauré ne vaut rien face au chaos des éléments. Le chaos (du grec $\chi \alpha \circ \varsigma)$ ), c'est d'abord l'ouverture béante, l'abîme, comme l'indique son étymologie ; il désigne chez Hésiode l'espace immense et ténébreux qui existait avant l'origine des choses, avant de désigner plus tardivement la masse confuse des éléments répandus dans

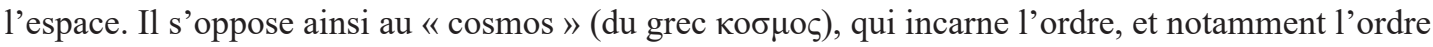
de l'univers, jusqu'à désigner le monde ou l'univers chez les Pythagoriciens.

12. Expression que Mahon emprunte à Wallace Stevens et à son célèbre poème « The Idea of Order at Key West »: « Oh ! Blessed rage for order, pale Ramon,/ The maker's rage to order words of the sea,/ Words of the fragrant portals, dimly-starred,/ And of ourselves and of our origins, /In ghostlier demarcations, keener sounds. » (Stevens, Collected Poems IIO-III).

13. On connaît la fascination de Mahon pour la poésie japonaise, notamment les haïkus de Bashô, ainsi que pour les $u k i y o-e$. 


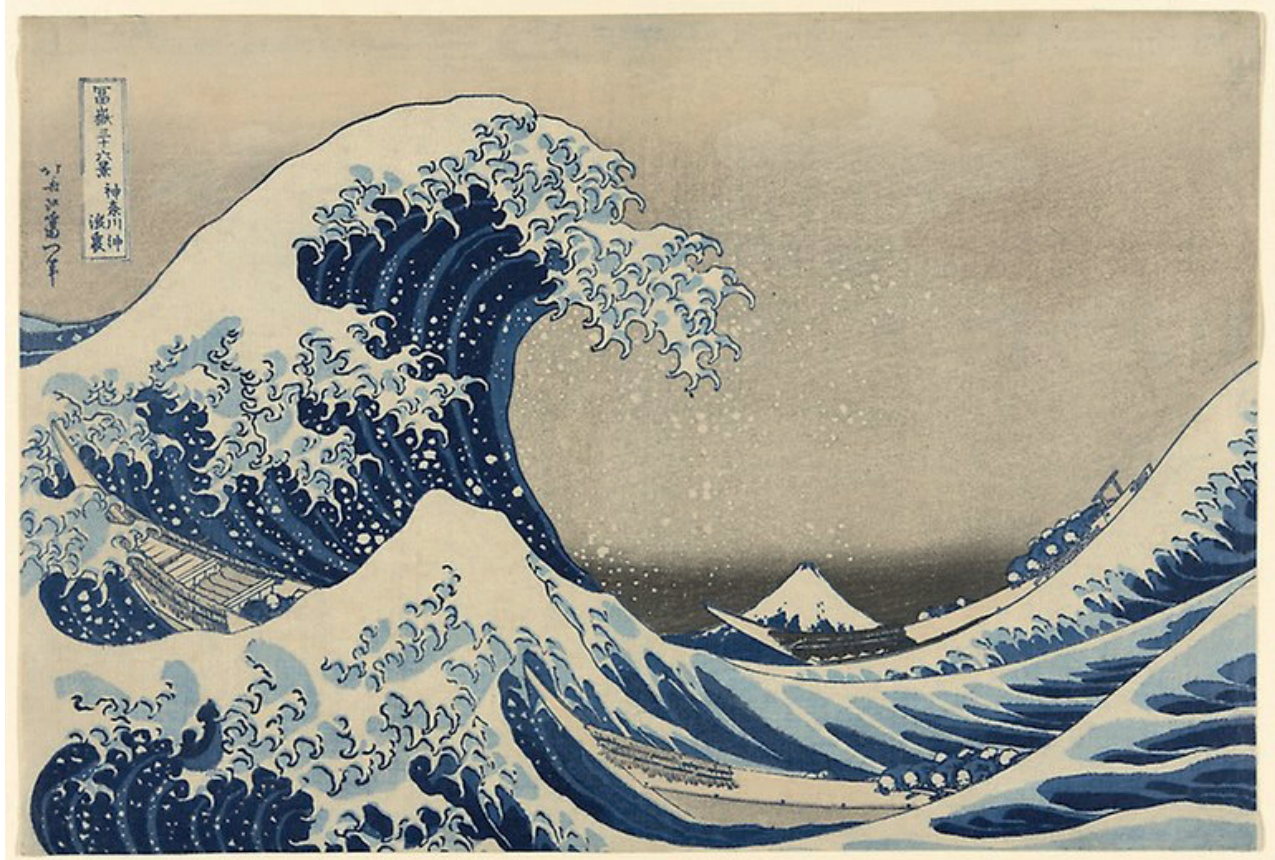

Figure 1. Katsushika Hokusai. Under the Wave off Kanagawa. 1826-1836. Estampe. Art Institute of Chicago. Public Domain. https://commons.wikimedia.org/wiki/File:Great_Wave_off_Kanagawa2.jpg

Mahon, avant d'être un poète de l'ordre des choses, se veut d'abord le poète du chaos, du moins y a-t-il une tentation chaotique dans sa poésie, présente dès la strophe fantôme de l'un de ses poèmes les plus emblématiques, intitulé «Courtyards in Delft », où il en appelle aux Ménades et à leur folie destructrice, afin de venir anéantir les germes de la violence contenus en puissance dans les arrière-cours trop propres de Delft et de Belfast :

If only, now, the Maenads, as of right,

Came smashing crockery, with fire and sword,

We could sleep easier in our beds at night.

(THBN IO) 


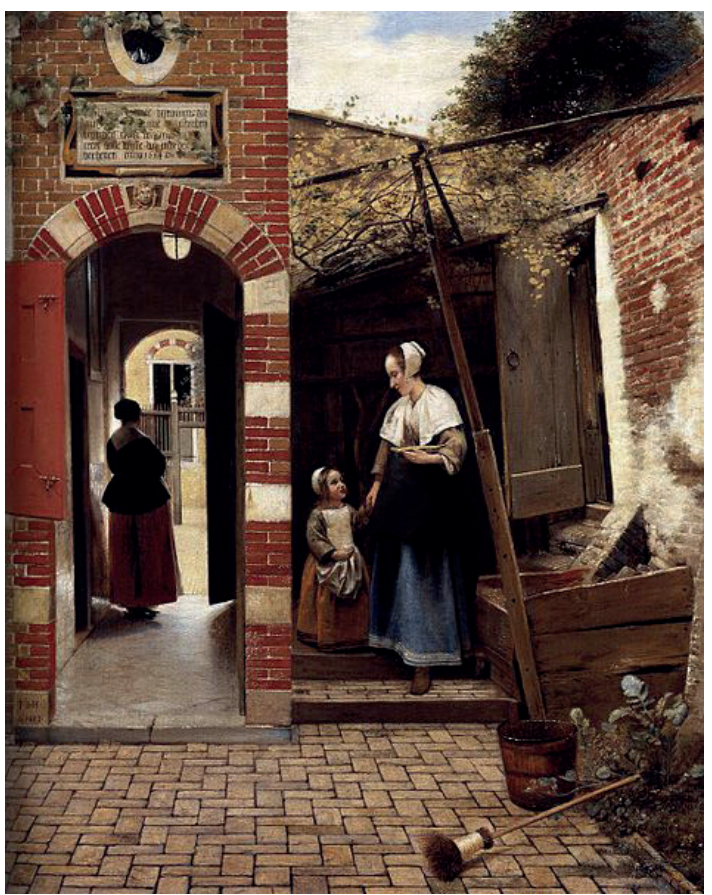

Figure 2. Pieter De Hooch. The Courtyard of a House in Delft. 1658. Huile. National Gallery,

Londres. Public Domain. https://commons. wikimedia.org/wiki/File:Pieter_de_Hooch_-_The_ Courtyard_of_a_House_in_Delft.jpg

Cette dernière strophe de « Courtyards in Delft » est la clef du poème, clef que cependant Mahon a jugé bon de supprimer dans des éditions postérieures ; ainsi cette strophe « fantôme » ne figure plus dans la première édition des Selected Poems de I99I. La jugeait-il trop explicite ? Ou était-il gêné par l'appel à la violence qui y était exprimé ? L'avant-dernière strophe de « Courtyards in Delft » nous laisse déjà sur l'impression d'un paysage dévasté, à venir pour les contemporains de Pieter de Hooch (Figure 2) ${ }^{14}$ et déjà là pour les contemporains de Mahon :

I lived there as a boy and know the coal Glittering in its shed, late-afternoon Lambency informing the deal table, The ceiling cradled in a radiant spoon. I must be lying low in a room there, A strange child with a taste for verse, While my hard-nosed companions dream of war On parched veldt and fields of rain-swept gorse; $[\ldots](T H B N$ 9)

« While my hard-nosed companions dream of fire/And sword upon parched veldt and fields of rain-swept gorse » : en l'espace d'un vers, Mahon relie la Hollande à l'Irlande du Nord, avec l'emploi de termes comme « veldt» et « gorse ». En effet, le mot « veldt» qui vient de l'afrikaans désigne la steppe de 1'Afrique du Sud, détail significatif qui renvoie à la politique coloniale de la Hollande au dix-septième siècle, que Mahon met en parallèle avec celle menée par Guillaume d'Orange en Irlande à la même époque, comme le suggère le mot « gorse », terme protestant pour le mot « whin », qui

14. Le poème « Courtyards in Delft » est un poème ekphrastique, faisant référence au célèbre tableau de Pieter de Hooch, The Courtyard of a House in Delft (I658), National Gallery, Londres (Figure 2). 
désigne une plante, l'ajonc (Scammell 6). Ces deux derniers vers préfigurent ainsi l'envers du décor de ces « Courtyards » de Delft en apparence si paisibles, si sereines, si proprettes, décor que Mahon va révéler dans la toute dernière strophe ${ }^{15}$ seulement. Que révèle donc cette strophe ? Elle révèle ainsi le visage caché de Delft (ou de la banlieue tranquille de Glengormley près de Belfast où Mahon a grandi), à savoir qu'elle n'est que la partie émergée de l'iceberg d'une stratégie commerciale et politique à vaste échelle, et qui ne fait que commencer, c'est-à-dire l'expansion démesurée de l'empire des Provinces-Unies au dix-septième siècle ${ }^{16}$ :

For the pale light of that provincial town

Will spread itself, like ink or oil,

Over the not yet accurate linen

Map of the world which occupies one wall

And punish nature in the name of God.

(THBN IO)

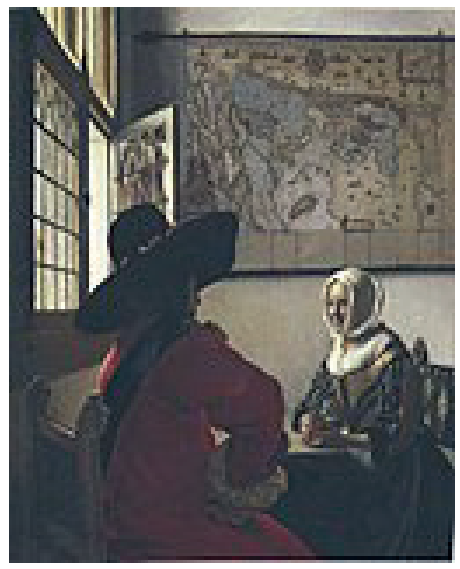

Figure 3. Johannes Vermeer. Officer and Laughing Girl. 1657. Huile. The Frick Collection, New York City. Public Domain. Wikimedia Commons. https://commons.wikimedia.org/wiki/File:1657_Vermeer_Officer_and_ Laughing_Girl_anagoria.jpg

15. Il y a d'ailleurs sans doute un effet voulu de la part de Mahon sur le plan de la mise en page, puisque dans l'édition de The Hunt by Night, où le poème est à cheval sur deux pages, il faut tourner la page pour découvrir la dernière strophe, et donc découvrir quel est l'envers du décor du tableau de Pieter de Hooch et du poème de Mahon.

16. Même si dans les années I650 les Provinces-Unies connaissent une relative stabilité, elles continuent d'étendre leur empire commercial, notamment avec la Compagnie néerlandaise des Indes orientales, fondée en I602, qui joue un rôle essentiel en forgeant un monopole commercial néerlandais avec l'Océan Indien et l'Extrême-Orient qui devait durer deux siècles, ainsi qu'avec la création de la Compagnie néerlandaise des Indes occidentales, qui établit en Amérique du Nord la colonie de Nouvelle-Hollande avec pour capitale la Nouvelle-Amsterdam, qui devait devenir New-York. 
La menace que renferme cet intérieur si paisible est dans cette carte du monde ${ }^{17}$ accrochée au mur, qui révèle la nature profondément mercantiliste et belliqueuse de ces deux sociétés que Mahon met en parallèle. Ce mercantilisme effréné, lié à un désir d'expansion de ce que Barthes nomme «l'empire des marchandises », caractéristique de la société hollandaise du dix-septième siècle (Barthes I964, 22), entraîne des guerres visant à conquérir de nouveaux territoires ou à défendre l'identité de ce type de société. Mahon en a vu et subi les conséquences, que ce soit par la connaissance historique et livresque de la Hollande du dix-septième ou par son enfance et son éducation à Belfast dans les années cinquante et soixante, jusqu'à l'éclatement du conflit des Troubles en Irlande du Nord. Puisqu'il refuse de s'impliquer directement dans ce conflit ou de prendre parti, à tout le moins peut-il envisager des solutions de nature poétique, mêmes si celles-ci peuvent sembler un peu radicales :

If only, now, the Maenads, as of right,

Came smashing crockery, with fire and sword,

We could sleep easier in our beds at night.

(THBN IO)

Dans la mythologie grecque, les Ménades (du grec $\mu$ aıvo $\mu \alpha$, qui signifie « délirer », " être furieux »), connues également sous le nom de Bacchantes chez les Romains, sont les accompagnatrices de Dionysos et l'escortent, vêtues de peaux de bêtes, jouant du tambourin et secouant leur thyrse, en proie au délire dionysiaque. Ivres la plupart du temps, elles ne font pas attention à leurs gestes et quand elles deviennent folles, elles n'ont aucune pitié, démembrent les voyageurs et mangent leur chair crue $^{18}$... Mahon « adapte » les pratiques des Ménades au dix-septième (« sword ») et au vingtième siècles («fire »), en suggérant qu'elles viennent briser le symbole de ce trop-plein de richesses acquises parfois par la violence : « If only, now, the Maenads, as of right, /Came smashing crockery, ${ }^{19}$ with fire and sword $[\ldots] »$. Cette dernière strophe, qui répond à la violence par la violence, a donc ensuite été retirée par Mahon lui-même, sans doute parce que cette image des Ménades faisait trop clairement écho à la situation en Irlande du Nord à ce moment-là, comme le fait remarquer Hugh Haughton dans son remarquable ouvrage intitulé The Poetry of Derek Mahon:

17. Mahon fait peut-être ici allusion à un tableau de Vermeer bien connu, Officer and Laughing Girl (c.I657), où derrière la jeune fille qui se tient assise se trouve une carte accrochée au mur, symbole de richesse, qui se trouve être une carte de la Hollande et de la Frise occidentale dessinée par Willem Blaeu juste après I620 (Figure 3).

18. Selon la légende elles auraient ainsi déchiqueté Orphée, jalouses de le voir rester fidèle à Eurydice.

19. Je souligne. 
Powerful as this protest against the rage for order is, Mahon must have had second thoughts about it. However oppressive the domestic order represented in the painting, the Maenads might represent something worse, especially in the context of the terror campaigns in Northern Ireland at the time. (Haughton I59)

Quoi qu'il en soit, la solution que propose Mahon est en accord avec ce que lui-même va pratiquer tout au long de sa carrière poétique, et ce qu'il pratiquait déjà étant enfant vis-à-vis de sa propre mère :

My mother stopped working when she got married. That's what they did then. She became a housewife. She had only her husband and an infant to look after, but she became a housewife and very house-proud in the obsessive way that a woman in that position is. It's almost a question of what else she had to do? She'd keep dusting and keep everything as bright as a new penny. Of course, this was a bit of a strain on the child, an irritant. In fact, with my mother, no harm to her, I think it was pathological. But since little boys are usually rougher than house-proud mothers, there were times I would deliberately do things to be infuriating - knock over a cup or something. (Interview avec Eamon Grennan I5I)

\section{Casser l'objet odipien : à la recherche de l'objet transitionnel}

La mère de Mahon, qualifiée de « very house-proud», n'est pas sans rappeler les femmes des artisans décrites dans «Courtyards in Delft », dont elle fait partie de manière générique: " House-proud, the wives/Of artisans pursue their thrifty lives/Among scrubbed yards, modest but adequate » (THBN 9). Réduite au rang de femme au foyer, Maisie Mahon se retourne vers l'espace intérieur de son foyer, qu'elle ne cesse d'astiquer frénétiquement pour meubler le vide, ce que ne supporte pas son fils : « In fact, with my mother, no harm to her, I think it was pathological» (Interview avec Eamon Grennan I5I). L'expression assez anodine de «no harm to her » est pour Mahon une façon de s'excuser presque de la liberté qu'il prend en qualifiant le cas de sa mère de pathologique. Mais on peut l'entendre également de façon plus psychanalytique. Mahon, ne pouvant pas ou ne voulant pas s'en prendre directement à sa mère (" no harm to her »), s'en est pris aux objets la représentant : «there were times I would deliberately do things to be infuriating - knock over a cup or something » (Interview avec Eamon Grennan I5I). L'enfant qu'est le poète à ce moment ne supporte pas cette activité visant constamment à entretenir ce que dans le poème «A Bangor Requiem » il nomme « your wise monkeys and 'Dresden' figurines, /your junk chinoiserie and coy pastoral scenes » (CP 260), ou encore « your ornaments and 
other breakable stuff» ( $C P$ 260), objets qu'il lui jette à la figure, au sens figuré du terme, dans le poème, qui est pourtant censé être un « requiem », une élégie à sa mère qui vient de mourir.

Cette attitude typiquement enfantine, qui consiste à casser de rage les objets familiers, prend ici une dimension particulière puisque le poète-enfant, au lieu de s'en prendre à n'importe quel objet, choisit délibérément de casser les objets qu'affectionne sa mère, beaux en apparence mais qui ne sont que du toc (« your junk chinoiserie ») et qu'il méprise : « and other breakable stuff ». L'adjectif « breakable » n'est pas anodin, car il est de fait très important que ces objets puissent être cassés, afin que l'enfant fasse l'expérience de la destruction, étape fondatrice dans l'éducation affective et psychologique de l'enfant, comme le montre Donald W. Winnicott dans son essai intitulé « Objet de l'usage d'un objet ». La pulsion de colère ou d'agressivité qui passe par la destruction permet de placer l'objet hors de soi, de l'externaliser, et si l'objet survit il devient alors utilisable puisqu'il ne disparaît pas :

Il n'y a pas de colère dans la destruction de l'objet à laquelle je me réfère ici, bien qu'on puisse dire qu'il y a de la joie quand l'objet survit. À partir de ce moment - ou suscité par cette phase - l'objet dans le fantasme est toujours en train d'être détruit. Cette qualité d' « être toujours en train d'être détruit » fait ressentir la réalité de l'objet qui survit comme tel, renforce la qualité du sentiment et contribue à l'établissement de la constance de l'objet. L'objet peut alors être utilisé. (Winnicott 2000, I30)

Le problème dans la pulsion destructrice de Mahon, telle qu'il a pu la pratiquer étant enfant ( « there were times I would deliberately do things to be infuriating - knock over a cup or something »), consiste précisément dans le fait qu'une fois la tasse cassée, la tasse n'existe plus, de même que lorsqu'un bébé mange il lui arrive de ne pas éprouver de satisfaction une fois le repas fini, comme le fait remarquer Laura Dethiville dans son ouvrage intitulé La clinique de Winnicott :

Devant le traumatisme dû à la satisfaction de son besoin, c'est-à-dire devant la perte du désir, l'enfant reste sans recours. [...] L'objet, pour lui, aura bel et bien disparu [...]. Il pensera donc l'avoir détruit. C'est cela, la « destruction de l'objet ». Il n'est plus là comme objet désiré, donc il n'est plus là du tout. À la place de la mère d'amour, il y a du vide. «Il y a un trou là où il y avait un corps complet plein de richesse » (Dethiville III $)^{20}$

Afin d'éviter le traumatisme lié à la disparition définitive de l'objet, qui ne serait pas supportable, Mahon va donc d'abord s'employer, dans ses poèmes, à détruire les objets, afin de mieux les

20. Laura Dethiville se réfère à l'article de Winnicott, « La position dépressive dans le développement affectif normal » (I969, 239). 
recycler ensuite, transformant les objets cassés en véritables « objets transitionnels », pour reprendre un concept majeur de Winnicott. Ces derniers vont lui permettre de trouver un espace de créativité propre et de surmonter la séparation d'avec la mère (qui est aussi une métaphore de la « mère-patrie » nord-irlandaise) en allant vers le monde extérieur, espace que Winnicott dans un autre texte intitulé « Objets transitionnels et phénomènes transitionnels : une étude de la première possession non-moi » (I95I), nomme « l'aire intermédiaire » :

L'aire intermédiaire à laquelle je me réfère est l'aire allouée à l'enfant qui se situe entre la créativité et la perception objective basée sur l'épreuve de réalité. (Winnicott 20I0,54)

L'objet transitionnel comme le « doudou » va ainsi permettre à l'enfant d'instaurer une relation affective qui lui donnera un premier avant-goût d'indépendance. Dans son rapport à l'objet, l'enfant peut développer son pouvoir de création puisque, lorsqu'il est manipulé, cet objet subit les tentatives de destruction auxquelles il doit survivre : «L'objet est affectueusement câliné, il est aussi aimé avec passion et mutilé » (37). Il est également vital que l'objet transitionnel reste le même et ne soit pas changé sauf si c'est l'enfant lui-même qui lui fait subir des changements. Par exemple, Winnicott précise un peu plus loin qu'un objet transitionnel comme le doudou ne doit pas être lavé :

La mère accepte qu'il devienne sale et même malodorant parce qu'elle sait qu'en le lavant elle créerait une interruption dans l'expérience du petit enfant, ce qui pourrait détruire la signification et la valeur de l'objet pour l'enfant. (Winnicott 20I0, 35)

Est-ce pour cette raison que Mahon a choisi durant toute une partie de sa carrière poétique de privilégier les objets au rebut ${ }^{21}$, abandonnés dans des lieux malpropres, parce qu'ils étaient sales ? La saleté même de ces objets brisés lui aurait alors permis de se constituer un espace poétique indépendant de sa mère, puisque par définition elle n'aurait pas été en mesure, pour une fois, d'exercer sa « ragefor-order ", c'est-à-dire de nettoyer ces objets au rebut : le balai et le seau d'eau sont impuissants dans l'espace protégé de la poésie. En nettoyant et lavant frénétiquement et quotidiennement tous ses objets, Maisie Mahon n'aurait-elle pas sans le savoir détruit chez son fils la possibilité d'établir un rapport affectif avec un objet en bon état ? Trop de propreté nuit, trop de propreté tue l'objet en lui enlevant sa substance, en le nettoyant de son âme ${ }^{22}$.

21. Sur la fonction quasi autoportraitique des objets au rebut dans la poésie de Mahon, se reporter à Naugrette-Fournier.

22. Le lien entre le désir de propreté et le désir de pureté morale a fait l'objet de plusieurs études, dont notamment celle de Mary Douglas dans son ouvrage fondateur intitulé Purity and Danger: An Analysis of Concepts of Pollution and Taboo. 


\section{Le grand nettoyage : repenser l'après tabula rasa}

Cependant, contrairement à ce qu'affirme Michael Longley dans un essai sur la poésie de l'Ulster (publié dans Causeway en I97I), où il décrit Mahon comme un " poète du feu et des airs " par opposition à Seamus Heaney, qu'il qualifie de « poète de la terre et de l'eau » (I07) ${ }^{23}$, l'eau reste très présente dans le monde pré ou post-apocalyptique des poèmes de Mahon. Si on trouve en effet de nombreuses références au feu dans la poésie de Mahon, comme dans " The Last of the Fire Kings » ${ }^{24}$, il nous semble cependant que la répartition des quatre éléments (à laquelle procède Longley) entre Mahon et Heaney mérite d'être nuancée, et que la poésie de Mahon est également une poésie de l'eau et de la terre. L'eau jouerait alors dans l'univers poétique de Mahon le rôle de la puissance destructrice, chargée de balayer et de « nettoyer » au sens ravageur du terme l'ordre des valeurs sociales, incarnées par les objets de consommation. Mahon, en voulant « nettoyer » la terre des hommes de leur matérialisme excessif, endosserait-il alors un rôle peu ou prou semblable à celui de sa mère, qui «nettoyait » son intérieur de manière frénétique, afin d'en enlever la moindre impureté ?

Si les méthodes sont comparables, la différence entre le fils et la mère réside justement dans le fait que l'entreprise de nettoyage de Maisie Mahon, décrite dans « Courtyards in Delft» et « A Bangor Requiem », participe à la préservation de cette société matérialiste que son fils critique et à laquelle il s'attaque dans ses poèmes, en invoquant les forces de la nature, et notamment l'eau, afin que celle-ci vienne détruire ces objets emblématiques de la société de consommation. Quant à la terre, si Longley décrit la poésie de Heaney comme «émergeant de processus de sédimentation », elle est également plus présente qu'on ne croit dans la poésie de Mahon, et même de plus en plus, et ce en particulier dans ce que Hugh Haughton nomme dans un article à son propos « the dialectic of rubbish » (Haughton 324). Au contraire, Mahon se penche sur les objets qui retournent à la terre, du moins en ce qui concerne les objets-déchets susceptibles de se dissoudre naturellement, à la différence des objets-déchets industriels qui, à défaut d'avoir été triés au préalable, resteront là où ils ont été abandonnés, sources de pollution de l'environnement. La terre, mêlée à l'eau, incarne dans la poésie de Mahon une force organique

23. «Seamus Heaney's talent complements interestingly that of Derek Mahon. Since both refer frequently in their work to the four elements, a comparison might be pursued by granting Heaney water and earth, Mahon fire and air. Or, to crystalise the notion in a geological analogy, Heaney's poetry emerges from the sedimentary processes, Mahon's from the igneous. » (Longley, I07). En I977, Heaney et Mahon ont d'ailleurs participé ensemble à une tournée de lectures de leurs poèmes en Irlande du Nord, tournée qui s'intitulait « In their Element », en référence à cette citation de Michael Longley et à cette répartition qu'il fait des quatre éléments entre les deux poètes.

24. «Last of the fire kings, I shall/Break with tradition and/Die by my own hand/Rather than perpetuate/The barbarous cycle» (Derek Mahon « The Last of the Fire Kings », CP 64-65). 
susceptible elle aussi de semer le chaos, en salissant et en déformant à tout jamais les objets qu'elle touche, comme dans «The Great Wave » où elle a tout emporté, révélant après son départ le désordre et la désolation lorsque la boue se retire :

The swirling mud receded leaving a waste of bodies, furniture, palm trunks, dereliction and in the streets the contents of an ocean. $(A A W 77)$

Tel un démiurge vengeur, Mahon éprouve un sentiment de satisfaction ambiguë lorsqu'il contemple, dans sa poésie, le Déluge qui s'abat régulièrement par le biais de catastrophes naturelles sur la planète, éprouvant naturellement un sentiment d'horreur face à cette destruction chaotique qu'il réprouve en fervent partisan de l'ordre, mais éprouvant également une certaine fascination face à cette puissance purificatrice de la nature (qui n'est pas sans rappeler ce que Burke dit en I757 du sublime dans A Philosophical Enquiry into the Origin of Our Ideas of the Sublime and Beautiful). La tabula rasa destructrice pratiquée par la nature permet ainsi de reconstruire puis de recycler sur des bases nouvelles pour ainsi révéler la nature cachée des objets de consommation et forcer le spectateur ou le lecteur à regarder la périphérie de l'innommable, à se pencher malgré lui sur ce qui constitue la lie de notre société, dans laquelle gît une source infinie de richesses à la fois économiques, écologiques et poétiques.

Car Mahon n'est pas pour autant adepte de la destruction totale, du « Ground Zero » après l'apocalypse. Le « Ground Zero » est cependant une étape indispensable du processus de création poétique mahonien, puisqu'il oblige à tout recommencer, à tout nettoyer. Ainsi, la saleté elle-même possède, dans les poèmes de Mahon, un aspect équivoque, car si elle se dépose partout, telle la lèpre, elle possède également un aspect quasi purificateur, puisqu'en cas de destruction massive elle provoque la nécessité de tout nettoyer, comme dans «After the Storm» :

A general cleaning-up operation began;

houses, garages, skips gleamed with the slime

deposited everywhere like a disease.

We will get over it though we're not sure how.

(AAW 29)

La saleté recouvre en même temps qu'elle désigne, puisque d'une certaine façon elle montre du doigt ce qui a été sali et qu'il faut donc changer. La destruction, puis la ruine seraient-elles donc 
deux étapes indispensables dans le renouveau poétique prôné par Mahon ? Le poète nord-irlandais aurait-il ainsi trouvé un remède à sa « rage for order », " the old curse » comme il la nomme lui-même dans « Under the Volcanoes » (New Collected Poems ${ }^{25} 363$ ), en acceptant la part de destruction qui est implicitement comprise dans toute notion d'ordre, et en la sublimant afin de reconstruire sur les débris un monde nouveau ? Car, ainsi qu'il le souligne dans son poème " As It Should Be », qui évoque un assassinat en Irlande du Nord, toute notion d'ordre contient une part d'exclusion et donc de destruction, ${ }^{26}$ comme c'est le cas de tous les totalitarismes et de tous les extrémismes :

We hunted the mad bastard

Through bog, moorland, rock, to the starlit west

And gunned him down in a blind yard

Between ten sleeping lorries

And an electricity generator.

[...]

This is as it should be.

They will thank us for it when they grow up

To a world with method in it.

(CP 49)

La « rage for order » est donc bien une malédiction comme le laisse entendre Mahon : elle implique une part incompressible de destruction qui apparente le poète aux siens, à ses « fire-loving people » («The Last of the Fire Kings », CP 65), qu'il réprouve et dont il nie pourtant le lien d'appartenance (d'où ce « we » qui laisse planer le doute sur la position du poète). De plus, ce penchant pour l'organisation et l'ordre qui caractérise le poète (et déjà sa mère auparavant) l'apparente d'une certaine manière à la logique délirante mais rigoureuse de ces extrémismes qu'il réprouve : le vers « to a world with method in it » n'est pas sans rappeler le « Though this be madness, yet there is method in't » de Hamlet. Mais au lieu de lui-même détruire, Mahon va trouver à son penchant pour la destruction une autre alternative, c'est-à-dire ramasser les débris des autres et les rassembler, les collectionner afin de leur redonner une autre forme, comme il le dit lui-même dans « Under the Volcanoes » contre les éléments hostiles, « imposing chaos where I try to wring/form from the debris choking up the mind » (NCP 363). La violence rentrée du poète s'exerce vis-à-vis du travail imposé par l'exigence de la « remise en forme » des ruines et des débris qui jonchent le paysage de la société.

25. Recueil que nous désignerons par la suite sous l'abréviation $N C P$.

26. Ce poème est d'ailleurs placé après le poème « Rage for Order » dans les Collected Poems, ce qui n'est pas un hasard. 
Le processus de destruction des objets s'avère donc fondamental et thérapeutique dans le cas de la relation de Mahon aux objets, puisqu'il lui permet à la fois de s'émanciper de l'hystérie maternelle de propreté et d'ordre et d'élaborer sa propre méthode de création poétique, qui implique une apocalypse, un meurtre symbolique pour retrouver les « raw materials » de ce monde, comme dans la suite du poème ekphrastique " 'Shapes and Shadows' " : ${ }^{27}$

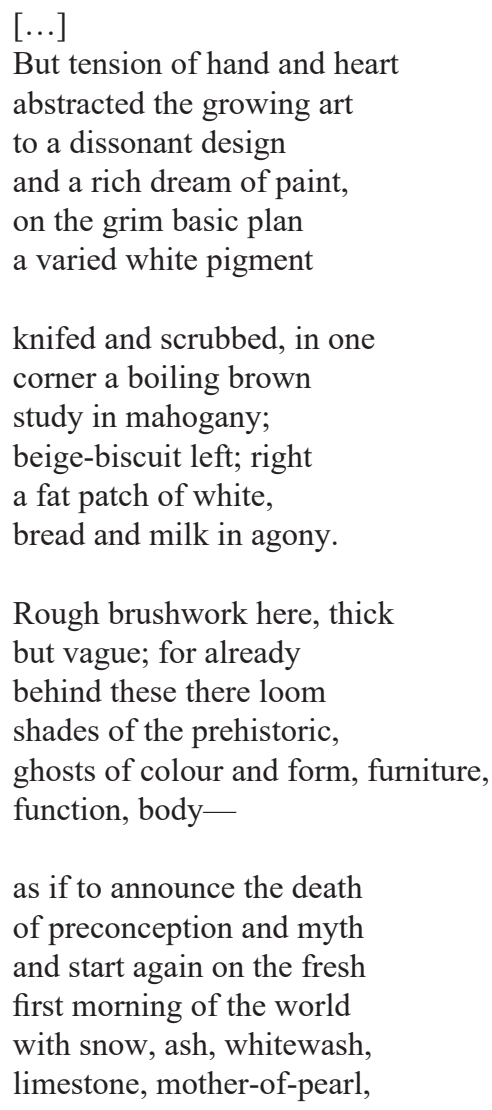

27. Le poème de Mahon fait référence au tableau du même nom, peint vers I962 par le peintre William Scott (I9I3-I989), tableau qui se trouve à l’Ulster Museum à Belfast. 
bleach, paper, soap and foam, top-of-the-milk cream, to find in the nitty-gritty of surfaces and utensils the shadow of a presence, a long-sought community. (CP 278-279)

Après le meurtre symbolique des objets sur la toile à l'aide d'un pigment brutalement appliqué ( « knifed and scrubbed »), un étalage grossier de couleur qui dissout les objets ( bread and milk in agony »), la création poétique peut commencer avec ce qu'il reste des objets détruits, c'est-à-dire les matières brutes : " as if to announce the death/of preconception and myth/and start again on the fresh/ first morning of the world/with snow, ash, whitewash,/limestone, mother-of-pearl [...] ». La nature morte hollandaise a disparu, place à la déconstruction et à l'abstraction figurative, afin de retrouver la substance de la chose au sein même de l'objet mis à nu, source de toute création artistique : « to find in the nitty-gritty/of surfaces and utensils/the shadow of a presence,/a long-sought community ».

\section{OUVRages CITÉS}

APPADURAI, Arjun. «Introduction: commodities and the politics of value ». The Social Life of Things: Commodities in cultural perspectives. Ed. Arjun Appadurai. Cambridge : Cambridge University Press, I986.

BARTHES, Roland. « Le monde-objet ». Essais Critiques. Paris : Éditions du Seuil, 1964.

BARTHES, Roland. Mythologies. Paris : Éditions du Seuil, I957.

BARTHES, Roland. « Réquichot et son corps ». L'Obvie et L'Obtus in Essais Critiques III. Paris : Éditions du Seuil, I982.

BURKE, Edmund. A Philosophical Enquiry into the Origin of Our Ideas of the Sublime and Beautiful [I757]. Oxford : Oxford University Press, 2015.

CAMPBELL, Matthew (ed.). The Cambridge Companion to Contemporary Irish Poetry. Cambridge : Cambridge University Press, 2003.

CLARK, Heather. The Ulster Renaissance: Poetry in Belfast 1962-1972. Oxford : Oxford University Press, 2006.

DETHIVILLE, Laura. La clinique de Winnicott. Paris : Éditions Campagne Première, 2013. 
DOUGLAS, Mary. Purity and Danger: An Analysis of Concepts of Pollution and Taboo. New York : Routledge, 1966.

GOUHIER, Jean. « La marge : entre rejet et intégration ». Le Déchet, le rebut, le rien. Dir. Jean-Claude Beaune. Seyssel : Editions Champ Vallon, 1999, 80-89.

GRENNAN, Eamon (interview with). « Derek Mahon: The Art of Poetry No.82 ». The Paris Review, $\mathrm{n}^{\circ} 54$, (Spring 2000). URL: https://www.theparisreview.org/interviews/732/the-art-of-poetry-no-82-derek-mahon

HAUGHTON, Hugh. «'The bright garbage on the incoming wave': Rubbish in the poetry of Derek Mahon ». Textual Practice I6:2 (2002), 323-343.

HAUGHTON, Hugh. The Poetry of Derek Mahon. Oxford : Oxford University Press, 2007.

HEANEY, Seamus. « Digging ». Death of a Naturalist. 1966. In Opened Ground: Poems 1966-1996. Londres : Faber and Faber, 1996.

LONGLEY, Michael. Causeway: The Arts in Ulster. Belfast : The Arts Council of Northern Ireland, I97I.

MAHON, Derek. Poems 1962-1978. Oxford : Oxford University Press, I979.

MAHON, Derek. The Hunt By Night. Oxford : Oxford University Press, 1982.

MAHON, Derek. Selected Poems. Oldcastle : The Gallery Press, I99I.

MAHON, Derek. Collected Poems. Oldcastle : The Gallery Press, I999.

MAHON, Derek. An Autumn Wind. Oldcastle : The Gallery Press, 2010.

MAHON, Derek. New Collected Poems. Oldcastle : The Gallery Press, $201 \mathrm{.}$

NAUGRETTE-FOURNIER, Marion. " 'Random Fragments of Autobiography': Objects of the Self in Derek Mahon's Poetry ». Études Irlandaises 43:I (20I8). URL : http://journals.openedition.org/etudesirlandaises/5480.

NAUGRETTE-FOURNIER, Marion et Maryvonne BOISSEAU. « Derek Mahon's Geopoetic Horizons ». Études Irlandaises 44:I (2019), URL : http://journals.openedition.org/etudesirlandaises/7208.

SCAMMELL, William. « Derek Mahon Interviewed ». Poetry Review 8I:2 (Summer I99I).

SIMMEL, Georg. The Philosophy of Money. Londres : Routledge, 1978.

STEVENS, Wallace. Collected Poems. Londres : Faber and Faber, 2006.

SWIFT, Jonathan. Poems selected by Derek Mahon. Londres : Faber and Faber, 2006.

THOMPSON, Michael. Rubbish Theory: The Creation and Destruction of Value. Oxford : Oxford University Press, 1979. 
WINNICOTT, Donald W. « La position dépressive dans le développement affectif normal ». I954. De la pédiatrie à la psychanalyse. Trad. J. Kalmanovitch. Paris : Payot, I969.

WINNICOTT, Donald W. « Objet de l'usage d'un objet». I968. La Crainte de l'effondrement et autres situations cliniques. Trad. J. Kalmanovitch et M. Gribinski. Paris : Gallimard, 2000.

WINNICOTT, Donald W. «Objets transitionnels et phénomènes transitionnels : une étude de la première possession non-moi ». I95I. Les objets transitionnels. Trad. J. Kalmanovitch, A. Stronck-Robert, M. Michelin et L. Rosaz. Paris : Payot, 2010.

\section{Euvres citées}

DE HOOCH, Pieter. The Courtyard of a House in Delft. I658. Huile. National Gallery, Londres. Public Domain.

HOKUSAI, Katsushika. Under the Wave off Kanagawa. I826-I836. Estampe. Art Institute of Chicago. Public Domain.

SCOTT, William. Shapes and Shadows. c.I962. Huile. Ulster Museum, Belfast.

VERMEER, Johannes. Officer and Laughing Girl. c.I657. Huile. Frick Collection, New York. Public Domain. 